CERN-PPE/96-031

March 7, 1996

\title{
A Study of Four-Fermion Final States with High Multiplicity at LEP
}

\author{
The OPAL Collaboration
}

\begin{abstract}
A search for $\ell^{+} \ell^{-} \mathrm{q} \overline{\mathrm{q}}(\ell=\mathrm{e}, \mu)$ four-fermion final states in high multiplicity events has been performed at $\sqrt{s} \approx m_{\mathrm{Z}}$ and above the $\mathrm{Z}$ peak at $\sqrt{s}=130 \mathrm{GeV}$ and $\sqrt{s}=136 \mathrm{GeV}$. The data taken with OPAL correspond to an integrated luminosity of $132.4 \mathrm{pb}^{-1}$ and $5.3 \mathrm{pb}^{-1}$ at $\sqrt{s} \approx m_{\mathrm{Z}}$ and $\sqrt{s}=130-$ $136 \mathrm{GeV}$, respectively. While at the $\mathrm{Z}$ resonance the data are in good agreement with the Standard Model predictions, we find more events than expected at $\sqrt{s}=130-136 \mathrm{GeV}$, especially in the $\mu^{+} \mu^{-} \mathrm{q} \overline{\mathrm{q}}$ channel, where 5 events remain after all cuts, with only 0.6 predicted by the Monte Carlo simulation. For all center-of-mass energies the observed shapes of the differential distributions are consistent with the predictions.
\end{abstract}

(Submitted to Physics Letters B) 
G. Alexander ${ }^{23}$, J. Allison ${ }^{16}$, N. Altekamp ${ }^{5}$, K. Ametewee ${ }^{25}$, K.J. Anderson ${ }^{9}$, S. Anderson ${ }^{12}$, S. Arcelli ${ }^{2}$, S. Asai ${ }^{24}$, D. Axen ${ }^{29}$, G. Azuelos ${ }^{18, a}$, A.H.Ball ${ }^{17}$, E.Barberio ${ }^{26}$, R.J. Barlow ${ }^{16}$, R. Bartoldus ${ }^{3}$, J.R. Batley ${ }^{5}$, G. Beaudoin ${ }^{18}$, J. Bechtluft ${ }^{14}$, C. Beeston ${ }^{16}$, T. Behnke ${ }^{8}$, A.N. Bell ${ }^{1}$, K.W.Bell ${ }^{20}$, G.Bella ${ }^{23}$, S. Bentvelsen ${ }^{8}$, P. Berlich ${ }^{10}$, S.Bethke ${ }^{14}$, O.Biebel ${ }^{14}$, V.Blobel ${ }^{8}$, I.J. Bloodworth ${ }^{1}$, J.E. Bloomer ${ }^{1}$, P. Bock ${ }^{11}$, H.M. Bosch ${ }^{11}$, M. Boutemeur ${ }^{18}$, B.T.Bouwens ${ }^{12}$,

S.Braibant ${ }^{12}$, R.M.Brown ${ }^{20}$, H.J. Burckhart ${ }^{8}$, C. Burgard ${ }^{27}$, R. Bürgin ${ }^{10}$, P. Capiluppi ${ }^{2}$, R.K. Carnegie ${ }^{6}$, A.A. Carter ${ }^{13}$, J.R. Carter ${ }^{5}$, C.Y. Chang ${ }^{17}$, C. Charlesworth ${ }^{6}$, D.G. Charlton ${ }^{1, b}$, D. Chrisman ${ }^{4}$, S.L. Chu ${ }^{4}$, P.E.L. Clarke ${ }^{15}$, I. Cohen ${ }^{23}$, J.E. Conboy ${ }^{15}$, O.C.Cooke ${ }^{16}$, M.Cuffiani ${ }^{2}$,

S. Dado ${ }^{22}$, C.Dallapiccola ${ }^{17}$, G.M.Dallavalle ${ }^{2}$, C.Darling ${ }^{31}$, S. De Jong ${ }^{12}$, L.A. del Pozo ${ }^{8}$, M.S.Dixit ${ }^{7}$, E. do Couto e Silva ${ }^{12}$, M.Doucet ${ }^{18}$, E.Duchovni ${ }^{26}$, G. Duckeck ${ }^{8}$, I.P. Duerdoth ${ }^{16}$, J.E.G.Edwards ${ }^{16}$, P.G.Estabrooks ${ }^{6}$, H.G. Evans ${ }^{9}$, M.Evans ${ }^{13}$, F.Fabbri ${ }^{2}$, P.Fath ${ }^{11}$, F. Fiedler ${ }^{12}$, M. Fierro ${ }^{2}$, H.M. Fischer ${ }^{3}$, R.Folman ${ }^{26}$, D.G. Fong ${ }^{17}$, M. Foucher ${ }^{17}$, H. Fukui ${ }^{24}$, A. Fürtjes ${ }^{8}$,

P. Gagnon ${ }^{7}$, A. Gaidot ${ }^{21}$, J.W. Gary ${ }^{4}$, J. Gascon ${ }^{18}$, S.M. Gascon-Shotkin ${ }^{17}$, N.I. Geddes ${ }^{20}$, C. Geich-Gimbel ${ }^{3}$, S.W. Gensler ${ }^{9}$, F.X. Gentit ${ }^{21}$, T. Geralis ${ }^{20}$, G. Giacomelli ${ }^{2}$, P. Giacomelli ${ }^{4}$, R. Giacomelli ${ }^{2}$, V. Gibson ${ }^{5}$, W.R. Gibson ${ }^{13}$, D.M. Gingrich ${ }^{30, a}$, J. Goldberg ${ }^{22}$, M.J. Goodrick ${ }^{5}$, W. Gorn ${ }^{4}$, C. Grandi ${ }^{2}$, E. Gross ${ }^{26}$, M. Gruwé ${ }^{8}$, C. Hajdu ${ }^{32}$, G.G.Hanson ${ }^{12}$, M. Hansroul ${ }^{8}$, M. Hapke ${ }^{13}$,

C.K.Hargrove ${ }^{7}$, P.A. Hart ${ }^{9}$, C.Hartmann ${ }^{3}$, M.Hauschild ${ }^{8}$, C.M.Hawkes ${ }^{5}$, R. Hawkings ${ }^{8}$, R.J.Hemingway ${ }^{6}$, G. Herten ${ }^{10}$, R.D.Heuer ${ }^{8}$, M.D.Hildreth ${ }^{8}$, J.C.Hill ${ }^{5}$, S.J. Hillier ${ }^{1}$, T. Hilse ${ }^{10}$, P.R.Hobson ${ }^{25}$, R.J.Homer ${ }^{1}$, A.K.Honma ${ }^{28, a}$, D. Horváth ${ }^{32, c}$, R. Howard ${ }^{29}$, R.E. Hughes-Jones ${ }^{16}$, D.E. Hutchcroft ${ }^{5}$, P. Igo-Kemenes ${ }^{11}$, D.C. Imrie ${ }^{25}$, M.R. Ingram $^{16}$, A. Jawahery ${ }^{17}$, P.W. Jeffreys ${ }^{20}$, H. Jeremie ${ }^{18}$, M. Jimack ${ }^{1}$, A. Joly ${ }^{18}$, G. Jones ${ }^{16}$, M. Jones ${ }^{6}$, R.W.L. Jones ${ }^{8}$, U. Jost ${ }^{11}$, P. Jovanovic ${ }^{1}$, J. Kanzaki ${ }^{24}$, D. Karlen ${ }^{6}$, T.Kawamoto ${ }^{24}$, R.K. Keeler ${ }^{28}$, R.G.Kellogg ${ }^{17}$, B.W.Kennedy ${ }^{20}$, J. King ${ }^{13}$,

J. Kirk ${ }^{29}$, S. Kluth ${ }^{8}$, T.Kobayashi ${ }^{24}$, M.Kobel ${ }^{10}$, D.S. Koetke ${ }^{6}$, T.P. Kokott ${ }^{3}$, S. Komamiya ${ }^{24}$,

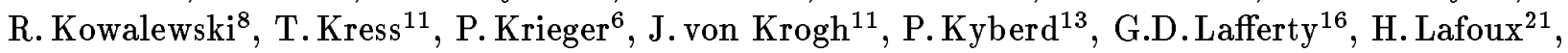
R. Lahmann ${ }^{17}$, W.P. Lai ${ }^{19}$, D. Lanske ${ }^{14}$, J. Lauber ${ }^{15}$, J.G. Layter ${ }^{4}$, A.M. Lee ${ }^{31}$, E. Lefebvre ${ }^{18}$, D. Lellouch ${ }^{26}$, J.Letts ${ }^{2}$, L.Levinson ${ }^{26}$, C.Lewis ${ }^{15}$, S.L.Lloyd ${ }^{13}$, F.K.Loebinger ${ }^{16}$, G.D. Long ${ }^{17}$, B. Lorazo ${ }^{18}$, M.J.Losty ${ }^{7}$, J. Ludwig ${ }^{10}$, A. Luig ${ }^{10}$, A. Malik ${ }^{21}$, M. Mannelli ${ }^{8}$, S. Marcellini $^{2}$, C.Markus ${ }^{3}$, A.J.Martin ${ }^{13}$, J.P. Martin ${ }^{18}$, G. Martinez ${ }^{17}$, T.Mashimo ${ }^{24}$, W. Matthews ${ }^{25}$, P. Mättig ${ }^{3}$, W.J. McDonald ${ }^{30}$, J. McKenna ${ }^{29}$, E.A.Mckigney ${ }^{15}$, T.J. McMahon ${ }^{1}$, A.I. McNab ${ }^{13}$, F. Meijers ${ }^{8}$, S. Menke ${ }^{3}$, F.S.Merritt ${ }^{9}$, H. Mes ${ }^{7}$, J.Meyer ${ }^{27}$, A.Michelini ${ }^{8}$, G.Mikenberg ${ }^{26}$, D.J.Miller ${ }^{15}$, R. Mir $^{26}$, W.Mohr ${ }^{10}$, A. Montanari ${ }^{2}$, T. Mori ${ }^{24}$, M. Morii ${ }^{24}$, U. Müller ${ }^{3}$, B. Nellen ${ }^{3}$, B. Nijjhar ${ }^{16}$, R. Nisius ${ }^{8}$, S.W. O'Neale ${ }^{1}$, F.G.Oakham ${ }^{7}$, F.Odorici ${ }^{2}$, H.O. ggren $^{12}$, T.Omori ${ }^{24}$, M.J. Oreglia ${ }^{9}$, S. Orito ${ }^{24}$, M.Palazzo ${ }^{2}$, J. Pálinkás ${ }^{33, d}$, J.P.Pansart ${ }^{21}$, G.Pásztor ${ }^{32}$, J.R.Pater ${ }^{16}$, G.N.Patrick ${ }^{20}$, M.J. Pearce ${ }^{1}$, S.Petzold ${ }^{27}$, J.E.Pilcher ${ }^{9}$, J.Pinfold ${ }^{30}$, D.E.Plane ${ }^{8}$, P.Poffenberger ${ }^{28}$, B.Poli ${ }^{2}$, A.Posthaus ${ }^{3}$, H.Przysiezniak ${ }^{30}$, D.L. Rees ${ }^{1}$, D. Rigby ${ }^{1}$, M.G. Rison ${ }^{5}$, S.A.Robins ${ }^{13}$, N. Rodning ${ }^{30}$, J.M. Roney ${ }^{28}$,

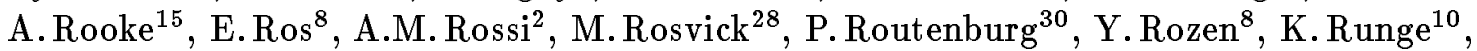
O.Runolfsson ${ }^{8}$, D.R.Rust ${ }^{12}$, R.Rylko ${ }^{25}$, E.K.G.Sarkisyan ${ }^{23}$, M.Sasaki ${ }^{24}$, C. Sbarra ${ }^{2}$, A.D.Schaile ${ }^{8, e}$, O.Schaile ${ }^{10}$, F.Scharf ${ }^{3}$, P.Scharff-Hansen ${ }^{8}$, P.Schenk ${ }^{4}$, B.Schmitt ${ }^{3}$, M. Schröder ${ }^{8}$, H.C.Schultz-Coulon ${ }^{10}$, M.Schulz ${ }^{8}$, P.Schütz ${ }^{3}$, J.Schwiening ${ }^{3}$, W.G.Scott ${ }^{20}$, T.G.Shears ${ }^{16}$, B.C.Shen ${ }^{4}$, C.H. Shepherd-Themistocleous ${ }^{27}$, P. Sherwood ${ }^{15}$, G.P.Siroli ${ }^{2}$, A.Sittler ${ }^{27}$, A.Skillman ${ }^{15}$, A.Skuja ${ }^{17}$, A.M.Smith ${ }^{8}$, T.J.Smith ${ }^{28}$, G.A.Snow ${ }^{17}$, R. Sobie ${ }^{28}$, S. Söldner-Rembold ${ }^{10}$,

R.W.Springer ${ }^{30}$, M.Sproston ${ }^{20}$, A.Stahl ${ }^{3}$, M.Starks ${ }^{12}$, K. Stephens ${ }^{16}$, J.Steuerer ${ }^{27}$, B.Stockhausen ${ }^{3}$,

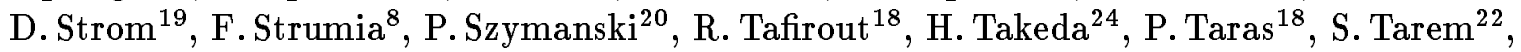
M. Tecchio ${ }^{8}$, N. Tesch ${ }^{3}$ M. Thiergen ${ }^{10}$, M.A. Thomson ${ }^{8}$, E. von Törne ${ }^{3}$, S. Towers ${ }^{6}$, M. Tscheulin ${ }^{10}$, E. $\operatorname{Tsur}^{23}$, A.S. Turcot ${ }^{9}$, M.F.Turner-Watson ${ }^{8}$, P.Utzat ${ }^{11}$, R. Van Kooten ${ }^{12}$, G. Vasseur ${ }^{21}$, M. Verzocchi ${ }^{10}$, P. Vikas ${ }^{18}$, M. Vincter ${ }^{28}$, E.H. Vokurka ${ }^{16}$, F. Wäckerle ${ }^{10}$, A. Wagner $^{27}$, C.P. Ward ${ }^{5}$, D.R. Ward ${ }^{5}$, J.J. Ward ${ }^{15}$, P.M. Watkins ${ }^{1}$, A.T. Watson ${ }^{1}$, N.K. Watson ${ }^{7}$, P. Weber ${ }^{6}$, P.S. Wells ${ }^{8}$, N. Wermes ${ }^{3}$, J.S. White ${ }^{28}$, B. Wilkens ${ }^{10}$, G.W.Wilson ${ }^{27}$, J.A. Wilson ${ }^{1}$, T. Wlodek ${ }^{26}$, G. Wolf ${ }^{26}$, 
S. Wotton ${ }^{11}$, T.R. Wyatt ${ }^{16}$, S. Xella ${ }^{2}$, S. Yamashita ${ }^{24}$, G. Yekutieli ${ }^{26}$, K. Yoshimura $^{24}$, V. Zacek ${ }^{18}$,

${ }^{1}$ School of Physics and Space Research, University of Birmingham, Birmingham B15 2TT, UK

${ }^{2}$ Dipartimento di Fisica dell' Università di Bologna and INFN, I-40126 Bologna, Italy

${ }^{3}$ Physikalisches Institut, Universität Bonn, D-53115 Bonn, Germany

${ }^{4}$ Department of Physics, University of California, Riverside CA 92521, USA

${ }^{5}$ Cavendish Laboratory, Cambridge CB3 0HE, UK

${ }^{6}$ Ottawa-Carleton Institute for Physics, Department of Physics, Carleton University, Ottawa, Ontario K1S 5B6, Canada

${ }^{7}$ Centre for Research in Particle Physics, Carleton University, Ottawa, Ontario K1S 5B6, Canada

${ }^{8}$ CERN, European Organisation for Particle Physics, CH-1211 Geneva 23, Switzerland

${ }^{9}$ Enrico Fermi Institute and Department of Physics, University of Chicago, Chicago IL 60637, USA

${ }^{10}$ Fakultät für Physik, Albert Ludwigs Universität, D-79104 Freiburg, Germany

${ }^{11}$ Physikalisches Institut, Universität Heidelberg, D-69120 Heidelberg, Germany

${ }^{12}$ Indiana University, Department of Physics, Swain Hall West 117, Bloomington IN 47405, USA

${ }^{13}$ Queen Mary and Westfield College, University of London, London E1 4NS, UK

${ }^{14}$ Technische Hochschule Aachen, III Physikalisches Institut, Sommerfeldstrasse 26-28, D-52056 Aachen, Germany

${ }^{15}$ University College London, London WC1E 6BT, UK

${ }^{16}$ Department of Physics, Schuster Laboratory, The University, Manchester M13 9PL, UK

${ }^{17}$ Department of Physics, University of Maryland, College Park, MD 20742, USA

${ }^{18}$ Laboratoire de Physique Nucléaire, Université de Montréal, Montréal, Quebec H3C 3J7, Canada

${ }^{19}$ University of Oregon, Department of Physics, Eugene OR 97403, USA

${ }^{20}$ Rutherford Appleton Laboratory, Chilton, Didcot, Oxfordshire OX11 0QX, UK

${ }^{21}$ CEA, DAPNIA/SPP, CE-Saclay, F-91191 Gif-sur-Yvette, France

${ }^{22}$ Department of Physics, Technion-Israel Institute of Technology, Haifa 32000, Israel

${ }^{23}$ Department of Physics and Astronomy, Tel Aviv University, Tel Aviv 69978, Israel

${ }^{24}$ International Centre for Elementary Particle Physics and Department of Physics, University of Tokyo, Tokyo 113, and Kobe University, Kobe 657, Japan

${ }^{25}$ Brunel University, Uxbridge, Middlesex UB8 3PH, UK

${ }^{26}$ Particle Physics Department, Weizmann Institute of Science, Rehovot 76100, Israel

${ }^{27}$ Universität Hamburg/DESY, II Institut für Experimental Physik, Notkestrasse 85, D-22607 Ham-

burg, Germany

${ }^{28}$ University of Victoria, Department of Physics, P O Box 3055, Victoria BC V8W 3P6, Canada

${ }^{29}$ University of British Columbia, Department of Physics, Vancouver BC V6T 1Z1, Canada

${ }^{30}$ University of Alberta, Department of Physics, Edmonton AB T6G 2J1, Canada

${ }^{31}$ Duke University, Dept of Physics, Durham, NC 27708-0305, USA

${ }^{32}$ Research Institute for Particle and Nuclear Physics, H-1525 Budapest, P O Box 49, Hungary

${ }^{33}$ Institute of Nuclear Research, H-4001 Debrecen, P O Box 51, Hungary

${ }^{a}$ and at TRIUMF, Vancouver, Canada V6T $2 \mathrm{~A} 3$

${ }^{b}$ and Royal Society University Research Fellow

${ }^{c}$ and Institute of Nuclear Research, Debrecen, Hungary

${ }^{d}$ and Depart of Experimental Physics, Lajos Kossuth University, Debrecen, Hungary

${ }^{e}$ and Ludwig-Maximilians-Universität, München, Germany 


\section{Introduction}

The electroweak Standard Model describes the production of two fermion-antifermion pairs in $\mathrm{e}^{+} \mathrm{e}^{-}$ collisions with small theoretical uncertainties. Below the $\mathrm{W}^{+} \mathrm{W}^{-}$threshold the only diagrams which contribute are those in which the fermion pairs emerge from neutral bosons, i.e. virtual photons and real or virtual $\mathrm{Z}$ bosons; in the case of electrons in the final state there are also contributions from $t$-channel boson exchange.

Four-fermion events have been studied in the past at various center-of-mass energies below [1] and on [2] the $\mathrm{Z}$ peak, with corresponding large variations in the relative contributions of the different diagrams. So far, no disagreement with the Standard Model predictions has been found within the experimental uncertainty for this rare process. The large number of events observed with the OPAL detector from 1990 to 1994 allows a test of the Standard Model predictions for four-fermion final states at the $\mathrm{Z}$ peak with high statistics. In addition, the recent energy upgrade of the LEP collider offers the first opportunity to study these processes at energies of $\sqrt{s}=130-136 \mathrm{GeV}$, well above the $\mathrm{Z}$ peak, where the relative contributions of the various Standard Model diagrams are again significantly changed.

A study of the four-fermion processes, particularly those containing a quark pair in the final state, is important because these channels form a sizeable background for Higgs searches. In addition, the good momentum resolution for lepton pairs in OPAL can be used to search for new particles $\mathrm{X}$ in the process $\mathrm{e}^{+} \mathrm{e}^{-} \rightarrow \gamma / \mathrm{Z}^{(*)} \mathrm{X} \rightarrow \ell^{+} \ell^{-} \mathrm{q} \overline{\mathrm{q}}(\ell=\mathrm{e}, \mu)$, where $\mathrm{X}$ or $\gamma / \mathrm{Z}^{(*)}$ decay to $\ell^{+} \ell^{-}$and $\mathrm{q} \overline{\mathrm{q}}$, respectively. We have analysed the OPAL data taken at $\sqrt{s} \approx m_{\mathrm{Z}}^{\dagger}$ from 1990 to 1994 with a total integrated luminosity of $132.4 \mathrm{pb}^{-1}$, searching for four-fermion events of the $\ell^{+} \ell^{-}$-jet-(jet) type, where the quark pair is detected as one or more jets of high multiplicity. A similar analysis was performed using all data with center-of-mass energies of $\sqrt{s}=130$ and $136 \mathrm{GeV}$ collected at the end of 1995 . The high energy data samples correspond to integrated luminosities of $2.7 \mathrm{pb}^{-1}$ and $2.6 \mathrm{pb}^{-1}$, respectively.

\section{The OPAL detector}

The OPAL detector is described in detail elsewhere [3]-[6]. It has a geometrical tracking acceptance of $98 \%$ of $4 \pi$ steradians. The central part consists of a set of tracking detectors in a $0.435 \mathrm{~T}$ solenoidal magnetic field. It is surrounded successively by a time-of-flight scintillation counter array, a lead-glass electromagnetic calorimeter with a presampler, an instrumented iron magnet yoke which serves as a hadron calorimeter, and finally by a set of muon chambers. The luminosity of the colliding beams is obtained from detecting small angle Bhabha scattering events in the two forward detectors.

\section{Monte Carlo sample}

To model the four-fermion signal events we use the FERMISV [7] program. The possible diagrams involving neutral boson exchange can be categorized into four different gauge-invariant groups (Fig. 1). The contribution of certain diagrams is especially high if the $\mathrm{Z}$ boson is on mass shell. Therefore, depending on the center-of-mass energy, different diagrams dominate the four-fermion cross section.

\footnotetext{
${ }^{\dagger}$ Most of the data were taken close to the peak of the $\mathrm{Z}$ resonance, with $\sqrt{s}=91.2 \mathrm{GeV}$. The off-peak samples, which correspond to about $20 \%$ of the total luminosity, are predominantly at $\sqrt{s}=89.4$ and $93.0 \mathrm{GeV}$.
} 
The FERMISV generator includes all the lowest order diagrams A-G, and their interferences. Initial and final state photon radiation from each fermion leg are modeled by convolving with an exponentiated radiation spectrum. The influence of initial state radiation on the cross sections is thereby reproduced at the percent level, whereas final state radiation, which barely influences the cross sections, is modeled to reproduce acceptance systematics with sufficient accuracy. The only electroweak corrections included are corrections to the $\mathrm{Z}$ boson vertices in the improved Born approximation. No higher order corrections are included in the photon propagator, nor for the $\gamma-q \bar{q}$ or $q-q \gamma$ vertices. We have estimated these corrections from basic principles and applied them as weights to the FERMISV prediction after the selection cuts. These weights are computed as a function of $m_{\min }=\min \left(m_{\ell \ell}, m_{\mathrm{qq}}\right)$, where $m_{\ell \ell}$ and $m_{\mathrm{qq}}$ are the invariant masses of the lepton and quark pair, respectively. We thereby assume that the system with the lower invariant mass has emerged from the virtual photon and the one with the larger mass from a $Z^{(*)}$. Misassignments due to intermediate $\gamma \gamma$ and $\mathrm{ZZ}$ are very rare and are neglected. In detail the corrections are as follows:

- Photon propagator: The running of $\alpha_{\mathrm{em}}\left(m_{\mathrm{min}}\right)$ was accounted for by explicitly summing the effect of leptonic loops, and taking the contribution due to hadronic loops from the parametrization of Ref. [8]. In addition, the effect from all $\mathrm{J} / \psi$ and $\Upsilon$ resonances in the propagator were summed, and appropriately taken into account. This correction applies to the six diagrams A-F. Depending on $m_{\min }$ the loop effects correct the cross section by up to $10 \%$. The resonant corrections to the total cross section are between $2 \%$ and $10 \%$ for low leptonic and hadronic masses, respectively.

- $\gamma$-q $\overline{\mathbf{q}}$ vertex: The QCD correction due to final state gluon radiation for this vertex is the same as that for the ratio $R_{\gamma}=\sigma\left(\mathrm{e}^{+} \mathrm{e}^{-} \rightarrow \gamma^{*} \rightarrow\right.$ hadrons $) / \sigma\left(\mathrm{e}^{+} \mathrm{e}^{-} \rightarrow \gamma^{*} \rightarrow \mu^{+} \mu^{-}\right)$. Down to invariant $q \bar{q}$ masses of $1 \mathrm{GeV}$ we include the first order correction $\alpha_{s}\left(m_{\min }\right) / \pi$, modified by a threshold term above the $c \bar{c}$ and $b \bar{b}$ threshold according to Ref. [9]. This correction applies to diagrams $B$, $\mathrm{D}, \mathrm{E}$, and F. The same correction was applied to the Z-q $\bar{q}$ vertex in diagrams $\mathrm{A}$ and $\mathrm{C}$.

- q-q $\gamma$ vertex: This vertex requires a large negative QCD correction for low photon energies as shown in Ref. [10] for on-shell photons, and should give similar effects for virtual photons. However, it applies only to diagram $A$, and therefore should have sizeable effects only at $\sqrt{s} \approx m_{\mathrm{Z}}$. Here we reweight the FERMISV predictions by a correction factor $\mathcal{C}\left(E_{\ell \ell}\right)$ depending on the lepton energy sum of each event. The function $\mathcal{C}\left(E_{\ell \ell}\right)$ was parametrized to reproduce approximately the suppression of real photon emission given in Ref. [10], varying from 1 for $E_{\ell \ell}>30 \mathrm{GeV}$ to 0.7 at $E_{\ell \ell}=10 \mathrm{GeV}$ and to 0.4 at $E_{\ell \ell}=3 \mathrm{GeV}$. For center-of-mass energies of 130 and $136 \mathrm{GeV}$ the contribution of diagram A to the total 'visible' cross section is small. In addition, our selection rejects events with low-energy virtual photons. We thus do not apply this correction and estimate the systematic error due to its omission to be less than $0.5 \%$.

With FERMISV we generated for each of the 10 possible $\ell^{+} \ell^{-} \mathrm{q} \overline{\mathrm{q}}$ final states Monte Carlo samples consisting of 2000, 200 and 200 events at $\sqrt{s}=91.2,89.4$ and $93.0 \mathrm{GeV}$, respectively, which corresponds to about 10 times the integrated luminosity recorded with OPAL from 1990 to 1994 . Furthermore, we generated one sample for each channel at a center-of-mass energy of $130 \mathrm{GeV}$ corresponding to an integrated luminosity of about $1000 \mathrm{pb}^{-1}$. Initial state radiation was enabled, and up to four final state photons were allowed to be radiated. The fragmentation of the two final state quarks was handled by JETSET 7.4 [11] with parameters tuned for multihadronic decays of the $Z^{0}$ as observed with the OPAL detector [12]. Possible variations of these parameters with the center-of-mass energy can be safely neglected, since the analysis does not impose stringent cuts on the hadronic system. In order to avoid generating events compatible with converted photons, which would then be rejected in the selection, the $\mathrm{e}^{+} \mathrm{e}^{-}$invariant mass in the final state was required to exceed $50 \mathrm{MeV}$. The momentum direction of each fermion was required to be more than $5^{\circ}$ away from the beam axis. The resulting 
(uncorrected) cross-sections for the various center-of-mass energies are listed in table 1. The variation in the contribution of the various subprocesses with $\sqrt{s}$ is obvious. Note that the electrons in the bremsstrahlung and multiperipheral classes are strongly forward peaked. Requiring all fermions to be generated at more than $15^{\circ}$, typically necessary for passing good charged track criteria, would reduce the generated bremsstrahlung and multiperipheral cross section by factors of 5 and 30, respectively. All events generated were processed by the OPAL detector simulation program [13]. For $\sqrt{s}=136 \mathrm{GeV}$ we scale the predictions from the $130 \mathrm{GeV}$ FERMISV Monte Carlo with the ratio of the cross sections at $\sqrt{s}=136$ and $130 \mathrm{GeV}$, assuming equal efficiencies.

The main physics background to the $\ell^{+} \ell^{-} q \bar{q}$ final state comes from multihadronic events with two relatively isolated leptons. These can arise either from semileptonic decays of heavy quarks, or in the case of electron pairs from a converted initial state photon. The latter mainly arises at center-of-mass energies above $\sqrt{s} \approx m_{\mathrm{Z}}$ and plays only a minor role at the $\mathrm{Z}$ resonance. To estimate this background we have analysed Monte Carlo events, simulated with JETSET 7.4. For $\sqrt{s}=130 \mathrm{GeV}$ and $136 \mathrm{GeV}$ we additionally used Monte Carlo samples generated with the PYTHIA 5.7 package [11], which also contain events of the type $\mathrm{e}^{+} \mathrm{e}^{-} \rightarrow(\gamma) \ell^{+} \ell^{-}$with $\ell=\mathrm{e}, \mu$ or $\tau$.

Background from other sources has been determined using KORALZ [14], BABAMC [15], FERMISV and EXCALIBUR [16]. The latter two were used to estimate possible background from all other fourfermion final states at $\sqrt{s} \approx m_{\mathrm{Z}}$ and $\sqrt{s}=130-136 \mathrm{GeV}$, respectively. The KORALZ and BABAMC Monte Carlo samples were analyzed to check the dilepton background at the $\mathrm{Z}$ peak. For $\sqrt{s}=130$ $136 \mathrm{GeV}$ these processes are included in the PYTHIA samples, with the exception of $t$-channel Bhabha events. Background from this channel again was estimated using an appropriate BABAMC Monte Carlo sample.

\section{Selection criteria}

In order to reduce the background from $\mathrm{e}^{+} \mathrm{e}^{-} \rightarrow \gamma / \mathrm{Z}^{(*)} \rightarrow$ hadrons one has to investigate a phase space region where four-fermion events have a clearly distinguishable signature compared to $q \bar{q}$-events. This is done by searching for isolated leptons with a certain minimum momentum. At $\sqrt{s} \approx m_{\mathrm{Z}}$ this leads to a rather low selection efficiency, since the cross section of the dominant diagram A peaks for low energy lepton pairs, which are emitted close to the jets. At $\sqrt{s}=130-136 \mathrm{GeV}$ the intermediate Zpole is reached in the conversion diagrams, where fermion pairs originating from the initial state have energies ranging typically from $32-35 \mathrm{GeV}$ for $\sqrt{s}=130 \mathrm{GeV}$ and from $36-39 \mathrm{GeV}$ for $\sqrt{s}=136 \mathrm{GeV}$. Due to this large energy of the radiated pair the leptonic and the hadronic system are in most cases clearly separated. Therefore, in this case searching for isolated leptons with rather high momenta selects $\ell^{+} \ell^{-} q \bar{q}$ events with much higher efficiencies and at the same time suppresses multihadronic background.

After removing cosmic ray events using time-of-flight and central tracking chamber information, a preselection of lepton pairs was performed, intended to yield a high statistics data sample for comparison with the Monte Carlo predictions. Since the preselected sample will later be used to determine the systematic error on our predicted background from $\mathrm{e}^{+} \mathrm{e}^{-} \rightarrow(\gamma) \mathrm{ff}$ events, we list in the following the detailed preselection criteria.

- At $\sqrt{s} \approx m_{\mathrm{Z}}$ we require the number of charged tracks per event $N_{\text {trk }} \geq 8$, where only tracks satisfying minimum quality criteria are considered. For the analysis at $\sqrt{s}=130-136 \mathrm{GeV}$ this requirement was loosened to $N_{\text {trk }} \geq 6$, since the background fraction coming from dilepton events with multiple photon conversions is strongly reduced off the $\mathrm{Z}$ resonance. 
- The number of jets per event has to be greater than or equal to two. Charged tracks and electromagnetic clusters not associated to any track are formed into jets using the Durham algorithm [17]. Typically one or two 'jets' originating from the leptons and in addition one or two jets originating from the quarks are expected for signal events.

- Leptons are identified differently for the analyses at $\sqrt{s} \approx m_{\mathrm{Z}}$ and at $\sqrt{s}=130-136 \mathrm{GeV}$. In both cases lepton candidates are required to have a momentum $p \geq 5 \mathrm{GeV} / c$. Since at the $\mathrm{Z}$ resonance the leptons are preferentially emitted close to the jets we employ a lepton identification optimized for b-tagging in a high-multiplicity environment. To select electrons we use an artificial neural network [18]. Electrons identified as originating from photon conversions are rejected [19]. Muons are identified as described in Ref. [19], mainly using the positional matching between the charged track extrapolation and the associated segment in the muon chambers. At $\sqrt{s}=130-$ $136 \mathrm{GeV}$ the leptons are expected to be isolated from the hadronic jets and we can therefore afford to apply much looser identification criteria. A track is classified as an electron if the ratio $E / p$ is greater than 0.8 , where $p$ is the track momentum and $E$ the associated electromagnetic energy. Furthermore the energy loss $\mathrm{d} E / \mathrm{d} x$ in the central tracking chamber has to be within a range of values where $99 \%$ of the electrons with this momentum are expected. Muons are required to have $E / p<0.2$ and at least three hits in the muon chambers or the last three layers of the hadronic calorimeter.

- For each lepton candidate an isolation angle $\alpha^{\text {iso }}$ is defined as the maximum angle at which the energy $E_{\text {cone }}$ contained within a cone of half-angle $\alpha^{\text {iso }}$ around the lepton is less than $1 \mathrm{GeV}$. In order to keep events with low-mass lepton pairs, $E_{\text {cone }}$ is defined to be the energy of all tracks and unassociated electromagnetic clusters within the cone, excluding both the lepton candidate itself, and also the nearest other lepton candidate if it lies within the cone.

The two most isolated leptons of each flavor are regarded as a candidate lepton pair. An event passes the preselection for $\mathrm{e}^{+} \mathrm{e}^{-} \mathrm{q} \overline{\mathrm{q}}$ or $\mu^{+} \mu^{-} \mathrm{q} \overline{\mathrm{q}}$, if a candidate lepton pair of the respective flavor is found. If the charges of the leptons are of the same sign, the event is rejected. Their momenta and isolation angles are called $p_{i}, i=1,2$, and $\alpha_{i}^{\text {iso }}$, respectively, where $i=1$ designates the lepton having the larger isolation angle. In the analysis at $\sqrt{s} \approx m_{\mathrm{Z}}$ we furthermore require at least one lepton with an isolation angle $\alpha^{\text {iso }}$ greater than $20^{\circ}$ to reduce the huge number of multihadronic events passing the criteria described above.

- In order to reject the few remaining dilepton events with multiple photon conversions we furthermore require that at least two charged tracks in the remaining hadronic system of an event have an energy loss inconsistent with an electron; here, the probability of the measured $\mathrm{d} E / \mathrm{d} x$ value being consistent with the energy loss of an electron inside the tracking chamber has to be less than $1 \%$.

After the preselection 171 and 136 events are found in the data at $\sqrt{s} \approx m_{\mathrm{Z}}$ and $\sqrt{s}=130-136 \mathrm{GeV}$, respectively. Figure 2 shows the isolation angle $\alpha_{1}^{\text {iso }}$ and the recoil mass distribution of the preselected events to be in good agreement with the expectation. The recoil mass $m_{\text {recoil }}$ is calculated using the momenta and energies of the two lepton tracks via $m_{\text {recoil }}^{2}=\left(1-2 E_{\ell \ell} / \sqrt{s}\right) s+m_{\ell \ell}^{2}$, where $E_{\ell \ell}$ is the leptonic energy and $m_{\ell \ell}$ is the invariant mass of the lepton pair candidate. The data are overlaid on the sum of the FERMISV prediction and the JETSET/PYTHIA background. Table 2 lists the number of expected and observed events separately for each channel and center-of-mass energy.

For the final selection we exploit the isolation of the signal leptons mentioned above, and further reject non-leptonic low momentum background by requiring

$$
\alpha_{1}^{\text {iso }} \geq 30^{\circ}, \alpha_{2}^{\text {iso }} \geq 10^{\circ} \text { and }\left|p_{1}\right|+\left|p_{2}\right| \geq 15 \mathrm{GeV} / c .
$$


In order to reject photon conversions for $\mathrm{e}^{+} \mathrm{e}^{-} \mathrm{q} \overline{\mathrm{q}}$ events we furthermore demand a transverse mass of the electron pair of more than $1 \mathrm{GeV}$. The transverse mass is calculated from the $\mathrm{e}^{+}$and $\mathrm{e}^{-}$ momenta projected onto a plane perpendicular to the beam axis. With these cuts we obtain from our Monte Carlo simulation total efficiencies for $\mathrm{e}^{+} \mathrm{e}^{-} \mathrm{q} \overline{\mathrm{q}}$ and $\mu^{+} \mu^{-} \mathrm{q} \overline{\mathrm{q}}$ events, respectively, $(1.0 \pm 0.1) \%$ and $(4.1 \pm 0.2 \%)$ at the $\mathrm{Z}$ resonance and $(4.0 \pm 0.4 \%)$ and $(18.5 \pm 1.2) \%$ at $\sqrt{s}=130-136 \mathrm{GeV}$.

\section{$5 \quad$ Results}

\subsection{Results at $\sqrt{s} \approx \mathrm{m}_{\mathrm{Z}}$}

With the selection criteria described above a total of 53 events are observed at $\sqrt{s} \approx m_{\mathrm{Z}}$, of which we find 28 in the $\mu^{+} \mu^{-} \mathrm{q} \overline{\mathrm{q}}$ final state and 25 in the $\mathrm{e}^{+} \mathrm{e}^{-} \mathrm{q} \overline{\mathrm{q}}$ channel. The predicted number of $\mu^{+} \mu^{-} \mathrm{q} \overline{\mathrm{q}}$ events from our corrected FERMISV Monte Carlo sample is $20.0 \pm 1.1$, while in the $\mathrm{e}^{+} \mathrm{e}^{-} \mathrm{q} \overline{\mathrm{q}}$ channel we expect $19.1 \pm 2.2$. After subtracting the expected multihadronic background in the $\mu^{+} \mu^{-} q \bar{q}\left(e^{+} e^{-} q \bar{q}\right)$ channel of $8.2 \pm 2.1\left(1.0_{-0.7}^{+1.3}\right)$ estimated from a JETSET Monte Carlo sample equivalent to about twice the integrated luminosity taken with OPAL from 1990 to 1994, the final number of four-fermion $\ell^{+} \ell^{-} \mathrm{q} \overline{\mathrm{q}}$ final states found in the data is $19.8 \pm 5.7$ and $24.0_{-5.0}^{+5.2}$ for the $\mu^{+} \mu^{-} \mathrm{q} \overline{\mathrm{q}}$ and $\mathrm{e}^{+} \mathrm{e}^{-} \mathrm{q} \overline{\mathrm{q}}$ channel, respectively. No background from lepton pairs is expected, due to the tight lepton identification criteria and the hard multiplicity cut. The total $\ell^{+} \ell^{-} \mathrm{q} \overline{\mathrm{q}}$ background from other four-fermion channels, such as $\mathrm{e}^{+} \mathrm{e}^{-} \tau^{+} \tau^{-}, \mu^{+} \mu^{-} \tau^{+} \tau^{-}$and $\tau^{+} \tau^{-} \tau^{+} \tau^{-}$, was estimated using FERMISV to be less than 0.5 events in total and is therefore negligible.

A possible background arises from color octet isolated quarkonium production from single hard gluon emission from quarks in multihadronic events, dominated by production of the $\mathrm{J} / \psi$ family, with a predicted branching ratio of $(3-4) \times 10^{-4}[20]$. This process was introduced recently to explain the excess of charmonium and bottomonium production at CDF [21]. It is also consistent with the recent OPAL observation of $\Upsilon$ production in $\mathrm{Z}^{0}$ decays [22]. An exact efficiency determination for selecting this process is difficult, since there exists no firm model for the amount and energy distribution of hadrons radiated from the color octet states when they turn into color singlet objects. Since the hard energy cut in the definition of the isolation angle is extremely sensitive to such hadrons, the efficiencies we derive from Monte Carlo simulations [22] have a large uncertainty for this process. In the mass regions of the $\mathrm{J} / \psi$ and $\Upsilon$ we find in total $6 \ell^{+} \ell^{-} \mathrm{q} \overline{\mathrm{q}}$ data events, while 4 events are predicted by the FERMISV and $\mathrm{e}^{+} \mathrm{e}^{-} \rightarrow(\gamma) \overline{f f}$ Monte Carlo. Since this suggests that our sensitivity to direct quarkonium production via color octet states is rather small, we neglect them in our final results.

\subsection{Results at $\sqrt{s}=130-136 \mathrm{GeV}$}

After applying all cuts $6 \ell^{+} \ell^{-} \mathrm{q} \overline{\mathrm{q}}$ events remain in the data sample taken at $\sqrt{s}=130-136 \mathrm{GeV}$, 5 in the $\mu^{+} \mu^{-} q \bar{q}$ channel and 1 in the $\mathrm{e}^{+} \mathrm{e}^{-} \mathrm{q} \overline{\mathrm{q}}$ channel. For these events we have listed in table 3 the leptonic and recoil masses $m_{\ell \ell}$ and $m_{\text {recoil }}$, the corresponding hadronic mass $m_{\text {had }}$, the isolation angle $\alpha_{1}^{\text {iso }}$ of the most isolated lepton candidate, $\left|\cos \theta_{\ell \ell}\right|$, where $\theta_{\ell \ell}$ is the angle between the lepton momentum sum and the beam axis, and the number of tracks per event $N_{\text {trk }}$. The hadronic mass $m_{\text {had }}$ is determined from all tracks and clusters of the recoiling hadronic system using the method of Ref. [23]. In the last column of the table, we have tentatively assigned each event to one of the diagrams in figure 1. Most of the events can easily be classified as examples of diagrams $\mathrm{C}$ or $\mathrm{D}$ according to whether the recoil or the leptonic mass is close to the $\mathrm{Z}$ mass. The event marked $\mathrm{C}(\mathrm{A})$ contains an isolated photon with $E_{\gamma}=30.8 \mathrm{GeV}$ and is therefore a candidate for a 'radiative return' 
annihilation process A with final state lepton pair production. However, since the lepton pair has a rather small $\theta_{\ell \ell}$, the photon is more likely to be from final state radiation. Therefore, the event is more typical of diagram $\mathrm{C}$. In the $\mathrm{e}^{+} \mathrm{e}^{-} \mathrm{q} \overline{\mathrm{q}}$ event neither the leptonic nor the recoil mass is consistent with the $\mathrm{Z}$ mass. This leads to the bremsstrahlung assignment, with diagram $\mathrm{F}$ preferred since the hadronic system in diagram $\mathrm{E}$ is more likely to be emitted in the forward direction. The various Monte Carlo predictions for the signal and background processes corresponding to an integrated luminosity of $5.3 \mathrm{pb}^{-1}$ are as follows.

Signal $\mathbf{e}^{+} \mathbf{e}^{-} \rightarrow \mathbf{e}^{+} \mathbf{e}^{-} \mathbf{q} \overline{\mathbf{q}}, \boldsymbol{\mu}^{+} \boldsymbol{\mu}^{-} \mathbf{q} \overline{\mathbf{q}}$. The FERMISV prediction is $\mathbf{0 . 5 5} \pm \mathbf{0 . 0 4}$ for $\boldsymbol{\mu}^{+} \boldsymbol{\mu}^{-} \mathrm{q} \overline{\mathrm{q}}$ and $0.63 \pm 0.06$ for $\mathrm{e}^{+} \mathrm{e}^{-} \mathrm{q} \overline{\mathrm{q}}$ events, giving a total of $1.18 \pm 0.07$ events. We have cross-checked this prediction against that of the EXCALIBUR program, where we find within the statistical uncertainty the same number of events predicted.

$\mathbf{e}^{+} \mathbf{e}^{-} \rightarrow(\gamma) \mathbf{f} \overline{\mathbf{f}}$. For the muon channel the background from multihadronic events or lepton pairs estimated with JETSET and PYTHIA is $0.06_{-0.03}^{+0.06}$ events. The corresponding estimated background for $\mathrm{e}^{+} \mathrm{e}^{-} \mathrm{q} \overline{\mathrm{q}}$ is $0.03_{-0.03}^{+0.07}$ events in our data sample.

Other four-fermion background. The misidentification of other 4-fermion events as $\mathrm{e}^{+} \mathrm{e}^{-} \mathrm{q} \overline{\mathrm{q}}$ and $\mu^{+} \mu^{-} \mathrm{q} \overline{\mathrm{q}}$ events has been estimated from non-signal events in the EXCALIBUR data sample. The predicted background from all other four-fermion channels was $0.06 \pm 0.02$ and $0.01 \pm 0.01$ events in our final data sample for $\mathrm{e}^{+} \mathrm{e}^{-} \mathrm{q} \overline{\mathrm{q}}$ and $\mu^{+} \mu^{-} \mathrm{q} \overline{\mathrm{q}}$, respectively.

Color octet quarkonium production. For the combined $\mu^{+} \mu^{-} q \bar{q}$ and $\mathrm{e}^{+} \mathrm{e}^{-} \mathrm{q} \overline{\mathrm{q}}$ channels less than 0.005 events are expected and therefore this process is neglected.

\section{$6 \quad$ Systematic Errors}

Three main sources of systematic uncertainties have to be considered:

1. Signal prediction. We assign the full higher order correction applied to the FERMISV Monte Carlo sample (section 3) as a systematic error. In certain phase space regions the corrections can reach values of up to $30 \%$, depending on the leptonic and hadronic masses. Integrated over the whole spectra this increases the FERMISV predictions for the $\mathrm{e}^{+} \mathrm{e}^{-} \mathrm{q} \overline{\mathrm{q}}$ and $\mu^{+} \mu^{-} \mathrm{q} \overline{\mathrm{q}}$ channel by $20.7 \%$ and $9.3 \%$ at the $\mathrm{Z}$ peak and $18.9 \%$ and $14.6 \%$ for $\sqrt{s}=130-136 \mathrm{GeV}$.

It is not necessary in this analysis to model the details of hadronic resonance production in the region below $m_{\text {recoil }}=3 \mathrm{GeV}$ for two reasons. Firstly, the multiplicity cut eliminates all resonances that decay into fewer than four charged particles $(\rho, \omega, \phi)$. Secondly, the Quark-Parton Model (QPM) prediction, used in FERMISV, produces a cross section that includes a rough average over all other resonances in this mass region. Since no mass cuts are applied to the hadronic system the systematic error from the omission of low-mass resonances is considered to be negligible.

2. $\mathbf{e}^{+} \mathbf{e}^{-} \rightarrow(\gamma) \mathbf{f f}$ background. Very few multihadronic background events pass the final selection cuts, according to the Monte Carlo prediction. It would thus be difficult to assess systematically the uncertainty on this background based on the final selection. We therefore use the higher statistics provided by the preselected event sample to estimate the systematic uncertainty on our 2-fermion background.

We compare data and Monte Carlo predictions for the size of the event increment, which we define as the number of events that pass the preselection cuts, but fail the final cuts. We take the statistical 
error on the increment added in quadrature with the difference of the increment between data and the JETSET/PYTHIA prediction as a conservative estimate of the systematic uncertainty. The resulting systematic errors are about $13 \%$ at $\sqrt{s} \approx m_{\mathrm{Z}}$ and $22 \%$ at $\sqrt{s}=130-136 \mathrm{GeV}$.

As an additional background cross-check we looked for e $\mu$ pairs, like-sign ee pairs, and like-sign $\mu \mu$ pairs after our preselection. Within our statistics we find in all cases reasonable agreement between data and Monte Carlo.

3. Four-fermion background. For the background estimation from other four-fermion events at $\sqrt{s}=130-136 \mathrm{GeV}$ using EXCALIBUR we conservatively assign a $50 \%$ systematic error. This should include all errors due to the neglect of the fermion masses and missing higher order corrections.

\section{Discussion}

\section{$7.1 \sqrt{s} \approx \mathrm{m}_{\mathrm{Z}}$}

Good agreement between the data and the Monte Carlo prediction is found in the shape and normalisation of the mass spectra of figure 3 . The final numerical results including all statistical and systematic errors are summarized in table 2 . For the $\mathrm{e}^{+} \mathrm{e}^{-} \mathrm{q} \overline{\mathrm{q}}$ and $\mu^{+} \mu^{-} \mathrm{q} \overline{\mathrm{q}}$ channel combined we find that after background subtraction there are $43.8_{-7.6}^{+7.7}$ four-fermion signal events. This should be compared with the FERMISV prediction of $39.1 \pm 5.6$ events. The number of events found at each center-of-mass energy around $m_{\mathrm{Z}}$ is consistent with expectations.

From the leptonic and hadronic mass distributions of the Monte Carlo prediction one finds that the expected contribution from the conversion diagrams $\mathrm{C}$ and $\mathrm{D}$ is negligible after all cuts. The lepton momenta cut $\left|p_{1}\right|+\left|p_{2}\right| \geq 15 \mathrm{GeV} / c$ equivalent to $m_{\text {recoil }}<\sqrt{s}-15 \mathrm{GeV}$ completely removes contributions from diagram $\mathrm{C}$. Diagram $\mathrm{D}$ is suppressed by the track multiplicity requirement, which tends to select high mass $q \bar{q}$ systems that rarely leave enough mass for the dilepton system to reach the $\mathrm{Z}$ peak. Thus the $\ell^{+} \ell^{-} \mathrm{q} \overline{\mathrm{q}}$ analysis at $\sqrt{s} \approx m_{\mathrm{Z}}$ shows agreement between measurement and prediction for the annihilation and bremsstrahlung processes, with an overall accuracy of $24 \%$. This accuracy is of about the same magnitude as the estimated higher order corrections that have been included in the prediction.

\section{$7.2 \sqrt{s}=130-136 \mathrm{GeV}$}

In the data at $\sqrt{s}=130-136 \mathrm{GeV}$ we find after background subtraction $5.8_{-2.4}^{+3.6}$ four-fermion signal events, for the $\mathrm{e}^{+} \mathrm{e}^{-} \mathrm{q} \overline{\mathrm{q}}$ and $\mu^{+} \mu^{-} \mathrm{q} \overline{\mathrm{q}}$ final state combined. This should be compared with the FERMISV prediction of $1.18 \pm 0.17$ events, as summarized in table 2 . The observed rate is thus a factor of $4.9_{-2.1}^{+3.1}$ larger than the prediction.

After the final selection the contribution of the conversion diagrams C and D is a factor of $20-30$ higher than the contribution from annihilation processes. For the $\mathrm{e}^{+} \mathrm{e}^{-} \mathrm{q} \overline{\mathrm{q}}$ channel we in addition have contributions from bremsstrahlung diagrams $\mathrm{E}$ and $\mathrm{F}$ of about the same magnitude as the conversion diagrams. In contrast to the situation at $\sqrt{s} \approx m_{\mathrm{Z}}$, at higher energies we therefore are sensitive only to the conversion diagrams and in the case of $\mathrm{e}^{+} \mathrm{e}^{-} \mathrm{q} \overline{\mathrm{q}}$ to the bremsstrahlung process.

If the observed excess of events were due to a leptonic or hadronic resonance, not present in the Monte Carlo simulations, one would expect peaks in either the leptonic invariant mass or the hadronic 
mass. The signal events of type $C$ are expected to peak at the $\mathrm{Z}$ mass in the hadronic spectrum and at corresponding low leptonic masses due to the virtual intermediate photon, and vice versa for type D. In figure $4 \mathrm{a}$ and $\mathrm{b}$ we have plotted the recoil mass versus the leptonic mass for data and the simulated signal events. No additional peak is seen in the data, but the events with large recoil masses cluster around the $\mathrm{Z}$ mass. From the kinematics of the events resonant states decaying into three fermions are highly disfavored, since these would not lead to final states where one of the leptonic or hadronic masses is compatible with the $\mathrm{Z}$ mass, while the other one is very small. We have furthermore searched for secondary vertices in the jets of the 6 remaining events, to examine the possibility of an enhancement of b-quarks [24]. No obvious b signature is observed in any of the jets.

A convenient classification is achieved by grouping together the observed and predicted events with high recoil mass $m_{\text {recoil }}>m_{\ell \ell}$ (mainly diagrams A and C) and with low recoil mass $m_{\text {recoil }}<m_{\ell \ell}$ (mainly diagrams B, D, E, and F). Following the same procedure to estimate backgrounds as above, we observe for high recoil masses no events in the $e^{+} e^{-} q \bar{q}$ channel, and 4 events in the $\mu^{+} \mu^{-} q \bar{q}$ channel, compared to $(0.30 \pm 0.04 \pm \mathbf{0 . 0 3})$ and $\left(0.51_{-0.04}^{+0.07} \pm 0.04\right)$ events, respectively, expected from the signal and background sources.

Table 4 summarizes the number of observed and expected events for the various sub-classes. For each sub-class the Poisson probability for observing at least the number of events in the data is given. The errors on the expected number of events are taken into account. The systematic errors have been taken to be $100 \%$ correlated between the sub-classes. The excess of data over the Monte Carlo prediction is more significant for the $\mu^{+} \mu^{-} \mathrm{q} \overline{\mathrm{q}}$ final state, and for events with high hadronic recoil mass.

In figure $4 \mathrm{c}-\mathrm{f}$ we compare various features of the events with the predicted distributions from FERMISV and background for $\mathrm{e}^{+} \mathrm{e}^{-} \mathrm{q} \overline{\mathrm{q}}$ and $\mu^{+} \mu^{-} \mathrm{q} \overline{\mathrm{q}}$. Even though the leptonic mass $m_{\ell \ell}$ in the data seems less sharply peaked towards zero mass than expected, no significant deviation from the expectation is observed in any of the distributions.

Since the kinematic properties seem very similar to the expectations a reasonable explanation for the observed excess of events is a statistical fluctuation. A bias of the selection towards the signal seems improbable, since the signal events lie generally far from all the cut values. Note that after all cuts the observed number of signal events in the $\mu^{+} \mu^{-} q \bar{q}$ final state corresponds to about twice the $\mu^{+} \mu^{-} q \bar{q}$ total cross section even before selection cuts as predicted by FERMISV, given in table 1.

\section{Conclusion}

We have performed a search for four-fermion final states of the topology $\ell^{+} \ell^{-}$-jet-(jet) at $\sqrt{s} \approx m_{\mathrm{Z}}$ and $\sqrt{s}=130-136 \mathrm{GeV}$. Different sub-processes dominate at the different center-of-mass energies. At the higher energies the contribution of the conversion diagrams becomes significant for the first time. At the $\mathrm{Z}$ resonance we observe $43.8_{-7.6}^{+7.7}$ events in good agreement with the Monte Carlo expectation of $39.1 \pm 5.6$. At $\sqrt{s}=130-136 \mathrm{GeV}$ we expect 1.18 signal and 0.16 background events for the $\mathrm{e}^{+} \mathrm{e}^{-} \mathrm{q} \overline{\mathrm{q}}$ and $\mu^{+} \mu^{-} \mathrm{q} \overline{\mathrm{q}}$ final states combined. We observe 6 events, 5 of them in the $\mu^{+} \mu^{-} \mathrm{q} \bar{q}$ channel, and 1 in the $\mathrm{e}^{+} \mathrm{e}^{-} \mathrm{q} \overline{\mathrm{q}}$ channel. No significant deviations in the shapes of the differential distributions from those predicted are observed. The Poisson probability for the observation of 6 events, where $1.3 \pm 0.2$ events are expected, is $0.3 \%$. 


\section{Acknowledgements:}

It is a pleasure to thank the SL Division for the efficient operation of the LEP accelerator, the precise information on the absolute energy, and their continuing close cooperation with our experimental group. In addition to the support staff at our own institutions we are pleased to acknowledge the Department of Energy, USA, National Science Foundation, USA, Particle Physics and Astronomy Research Council, UK, Natural Sciences and Engineering Research Council, Canada, Israel Ministry of Science, Israel Science Foundation, administered by the Israel Academy of Science and Humanities, Minerva Gesellschaft, Japanese Ministry of Education, Science and Culture (the Monbusho) and a grant under the Monbusho International Science Research Program, German Israeli Bi-national Science Foundation (GIF), Direction des Sciences de la Matière du Commissariat à l'Energie Atomique, France, Bundesministerium für Bildung, Wissenschaft, Forschung und Technologie, Germany, National Research Council of Canada, Hungarian Foundation for Scientific Research, OTKA T-016660, and OTKA F-015089. 


\section{References}

[1] See for example:

CELLO Collaboration, H. Behrend et al., Z. Phys. C43 (1989) 1;

M. Petradza et al., Phys. Rev. D42 (1990) 2171;

Mark II Collaboration, T. Barklow et al., Phys. Rev. Lett. B68 (1992) 13.

[2] OPAL Collaboration, P.D. Acton et al., Phys. Lett. B287 (1992) 369;

DELPHI Collaboration, P. Abreu et al., Nucl. Phys. B403 (1993) 3;

L3 Collaboration, A. Adam et al., Phys. Lett. B321 (1994) 283;

ALEPH Collaboration, D. Buskulic et al., Z. Phys. C66 (1995) 3.

[3] OPAL Collaboration, K. Ahmet et al., Nucl. Instrum. Methods A305 (1991) 275.

[4] P.P. Allport et al., Nucl. Instrum. Methods A324 (1993) 34

[5] P.P. Allport et al., Nucl. Instrum. Methods A346 (1994) 476

[6] B.E. Anderson et al., IEEE Transactions on Nuclear Science, 41 (1994) 845

[7] J. Hilgart, R. Kleiss and F. Le Diberder, Comp. Phys. Comm. 75 (1993) 191.

[8] H. Burkhardt, F. Jegerlehner, G. Penso, and C. Verzegnassi, Z.Phys. C43 (1989) 497.

[9] J. Schwinger: "Particles, Sources, and Fields", Addison-Wesley, New York, 1970;

R. Marshall, Z. Phys. C43 (1989) 595.

[10] P. Mättig, R. Spiesberger, W. Zeuner, Z. Phys. C60 (1993) 613.

[11] T. Sjöstrand, Comp. Phys. Comm. 82 (1994) 74.

[12] OPAL Collaboration, G. Alexander et al., CERN-PPE/95-126, to be published in Z. Phys. C.

[13] J. Allison et al., Nucl. Instrum. Methods A317 (1992) 47.

[14] S. Jadach, Z. Wąs, B.F.L. Ward, Comp. Phys. Comm. 66 (1991) 276.

S. Jadach, Z. Wạs, B.F.L. Ward, Comp. Phys. Comm. 79 (1994) 503.

[15] M. Boehm, A. Denner and W. Hollik, Nucl. Phys. B304 (1988) 687;

F.A. Berends, R. Kleiss and W. Hollik, Nucl. Phys. B304 (1988) 712.

[16] F.A. Berends and R. Pittau, Lorentz Institut Leiden, INLO-PUB 12/91 (1991).

[17] N. Brown and W.J. Stirling, Z. Phys. C53 (1992) 629.

[18] OPAL Collaboration, R. Akers et al., Phys. Lett. B327 (1994) 411.

[19] OPAL Collaboration, P.D. Acton et al., Z. Phys. C58 (1993) 523.

[20] P. Cho, Phys. Lett. B368 (1996) 171.

[21] CDF Collaboration, F. Abe et al., Phys. Rev. Lett. 75 (1995) 4358.

[22] OPAL Collaboration,G. Alexander et al., CERN-PPE/95-181, to be published in Phys. Lett. B.

[23] OPAL Collaboration, M.Z. Akrawy et al., Phys. Lett. B253 (1991) 511.

[24] OPAL Collaboration, R. Akers et al., Z. Phys. C65 (1995) 17. 


\begin{tabular}{|l|l||c|c|c||c|}
\hline \multirow{2}{*}{ Channel } & \multirow{2}{*}{ Diagram } & \multicolumn{3}{c|}{ Generated cross sections in pb at different values of $\sqrt{s}$} \\
\cline { 3 - 6 } & & $89.4 \mathrm{GeV}$ & $91.2 \mathrm{GeV}$ & $93.0 \mathrm{GeV}$ & $130.0 \mathrm{GeV}$ \\
\hline $\mathrm{e}^{+} \mathrm{e}^{-} \mathrm{q} \overline{\mathrm{q}}$ & Annihilation & 1.52 & 4.95 & 2.37 & 0.06 \\
& Conversion & 1.19 & 3.66 & 5.34 & 0.70 \\
& Bremsstrahlung & 2.34 & 2.34 & 2.21 & 1.56 \\
& Multiperipheral & 0.78 & 0.78 & 0.75 & 0.41 \\
\hline & Total & 5.91 & 11.69 & 10.43 & 2.58 \\
\hline \hline$\mu^{+} \mu^{-} \mathrm{q} \overline{\mathrm{q}}$ & Annihilation & 0.85 & 2.77 & 1.34 & 0.04 \\
& Conversion & 0.38 & 0.96 & 1.95 & 0.49 \\
\hline & Total & 1.22 & 3.73 & 3.29 & 0.53 \\
\hline
\end{tabular}

Table 1: FERMISV cross sections in $\mathrm{pb}$ for $\sqrt{s}=89.4,91.2,93.0$ and $130 \mathrm{GeV}$ requiring all final state fermions to be more than $5^{\circ}$ away from the beam axis, and an invariant mass for the $\mathrm{e}^{+} \mathrm{e}^{-}$pairs of more than $50 \mathrm{MeV}$. The total cross section includes interference effects and is therefore not the sum of the separate contributions. The statistical accuracy is about $1-3 \%$.

\begin{tabular}{|c|c|c|c|c|}
\hline & & $\mathrm{e}^{+} \mathrm{e}^{-} \mathrm{q} \overline{\mathrm{q}}$ Channel & $\mu^{+} \mu^{-} \mathrm{q} \overline{\mathrm{q}}$ Channel & Total \\
\hline & & \multicolumn{3}{|c|}{$\sqrt{s} \approx m_{\mathrm{Z}}$} \\
\hline \multirow[t]{4}{*}{ Preselection } & Signal & 32.6 & 26.5 & 59.1 \\
\hline & $\mathrm{e}^{+} \mathrm{e}^{-} \rightarrow(\gamma) \mathrm{ff}$ & 50.4 & 57.6 & 108.0 \\
\hline & Total expected & 83.0 & 84.1 & 167.1 \\
\hline & Total observed & 88 & 83 & 171 \\
\hline \multirow[t]{5}{*}{ Final selection } & Signal & $19.1_{-2.2}^{+2.2} \pm 3.3$ & $20.0_{-1.1}^{+1.1} \pm 1.7$ & $39.1_{-2.5}^{+2.5} \pm 5.0$ \\
\hline & $\mathrm{e}^{+} \mathrm{e}^{-} \rightarrow(\gamma) \overline{\mathrm{ff}}$ & $1.0_{-0.7}^{+1.3} \pm 0.1$ & $8.2_{-2.1}^{+2.1} \pm 1.1$ & $9.2_{-2.2}^{+2.5} \pm 1.2$ \\
\hline & Total expected & $20.1_{-2.3}^{+2.6} \pm 3.3$ & $28.2_{-2.4}^{+2.4} \pm 2.0$ & $48.3_{-3.3}^{+3.5} \pm 5.3$ \\
\hline & Total observed & 25 & 28 & 53 \\
\hline & & \multicolumn{3}{|c|}{$\sqrt{s}=130-136 \mathrm{GeV}$} \\
\hline \multirow[t]{4}{*}{ Preselection } & Signal & 1.7 & 0.8 & 2.5 \\
\hline & $\mathrm{e}^{+} \mathrm{e}^{-} \rightarrow(\gamma) \mathrm{ff}$ & 131.8 & 17.9 & 149.7 \\
\hline & Total expected & 133.5 & 18.7 & 152.2 \\
\hline & Total observed & 113 & 23 & 136 \\
\hline \multirow[t]{5}{*}{ Final selection } & Signal & $\overline{0.63_{-0.06}^{+0.06} \pm 0.10}$ & $\overline{0.55_{-0.04}^{+0.04} \pm 0.07}$ & 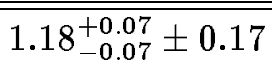 \\
\hline & $\mathrm{e}^{+} \mathrm{e}^{-} \rightarrow(\gamma) \overline{f f}$ & $0.03_{-0.03}^{+0.07} \pm 0.01$ & $0.06_{-0.03}^{+0.06} \pm 0.01$ & $0.09_{-0.04}^{+0.07} \pm 0.02$ \\
\hline & 4-fermion background & $0.06_{-0.02}^{+0.02} \pm 0.03$ & $0.01_{-0.01}^{+0.01} \pm 0.01$ & $0.07_{-0.02}^{+0.02} \pm 0.04$ \\
\hline & Total expected & $0.72_{-0.07}^{+0.09} \pm 0.10$ & $0.62_{-0.05}^{+0.07} \pm 0.07$ & $1.34_{-0.08}^{+0.02} \pm 0.17$ \\
\hline & Total observed & 1 & 5 & 6 \\
\hline
\end{tabular}

Table 2: Number of expected and observed events after the preselection and the final selection at $\sqrt{s} \approx m_{\mathrm{Z}}$ and $\sqrt{s}=130-136 \mathrm{GeV}$. For the final results both the statistical and the systematic errors are given; the statistical uncertainties on the signal and background prediction after the preselection are much smaller than the statistical error on the number of observed events. 


\begin{tabular}{|c||c|c|c|c|c|c|c|c|}
\hline Channel & $\sqrt{s}$ & $m_{\ell \ell}$ & $m_{\text {recoil }}$ & $m_{\text {had }}$ & $\alpha_{1}^{\text {iso }}$ & $\mid \cos \theta_{\ell \ell}$ & $N_{\text {trk }}$ & Class \\
\hline$\mu^{+} \mu^{-} \mathrm{q} \overline{\mathrm{q}}$ & 130 & $86.6 \pm 4.2$ & $18.3 \pm 26.0$ & $1.6 \pm 1.0$ & 113 & 0.157 & 6 & $\mathrm{D}$ \\
& 130 & $4.1 \pm 0.1$ & $95.0 \pm 0.8$ & $91.6 \pm 9.6$ & 66 & 0.810 & 16 & $\mathrm{C}$ \\
& 130 & $2.3 \pm 0.1$ & $94.7 \pm 0.8$ & $90.9 \pm 9.6$ & 34 & 0.827 & 15 & $\mathrm{C}(\mathrm{A})$ \\
& 136 & $0.4 \pm 0.1$ & $98.4 \pm 0.7$ & $94.9 \pm 9.6$ & 54 & 0.213 & 23 & $\mathrm{C}$ \\
& 136 & $3.5 \pm 0.1$ & $88.2 \pm 1.0$ & $97.8 \pm 9.6$ & 65 & 0.301 & 20 & $\mathrm{C}$ \\
\hline $\mathrm{e}^{+} \mathrm{e}^{-} \mathrm{q} \overline{\mathrm{q}}$ & 130 & $47.9 \pm 4.0$ & $10.5 \pm 10.6$ & $7.6 \pm 2.0$ & 122 & 0.248 & 10 & $\mathrm{~F}(\mathrm{E})$ \\
\hline
\end{tabular}

Table 3: Properties of remaining events at $\sqrt{s}=130-136 \mathrm{GeV}$ after all cuts. Given are the leptonic and the recoil mass $m_{\ell \ell}$ and $m_{\text {recoil }}$, the corresponding hadronic mass $m_{\text {had }}$, calculated using tracks and clusters of the recoiling hadronic system, the isolation angle $\alpha_{1}^{\text {iso }}$ of the most isolated lepton candidate, $\left|\cos \theta_{\ell \ell}\right|$, where $\theta_{\ell \ell}$ is the angle between the lepton momentum sum and the beam axis, and the number of tracks per event $N_{\text {trk. }}$. The units for $\sqrt{s}, m_{\ell \ell}, m_{\text {recoil }}$ and $m_{\text {had }}$ are $\mathrm{GeV}, \alpha_{1}^{\text {iso }}$ is given in degrees.

\begin{tabular}{|c|c|c|c|}
\hline & observed & expected & probability \\
\hline $\mathrm{e}^{+} \mathrm{e}^{-} \mathrm{q} \overline{\mathrm{q}}$ & 1 & $0.72_{-0.07}^{+0.09} \pm 0.10$ & $51.2 \%$ \\
$\mu^{+} \mu^{-} \mathrm{q} \overline{\mathrm{q}}$ & 5 & $0.62_{-0.05}^{+0.07} \pm 0.07$ & $0.06 \%$ \\
low recoil mass & 2 & $0.53_{-0.06}^{+0.09} \pm 0.10$ & $10.4 \%$ \\
high recoil mass & 4 & $0.81_{-0.06}^{+0.08} \pm 0.07$ & $1.0 \%$ \\
\hline total & 6 & $1.34_{-0.08}^{+0.10} \pm 0.17$ & $0.3 \%$ \\
\hline
\end{tabular}

Table 4: Number of expected and observed events at $\sqrt{s}=130-136 \mathrm{GeV}$ and the corresponding probabilities. 

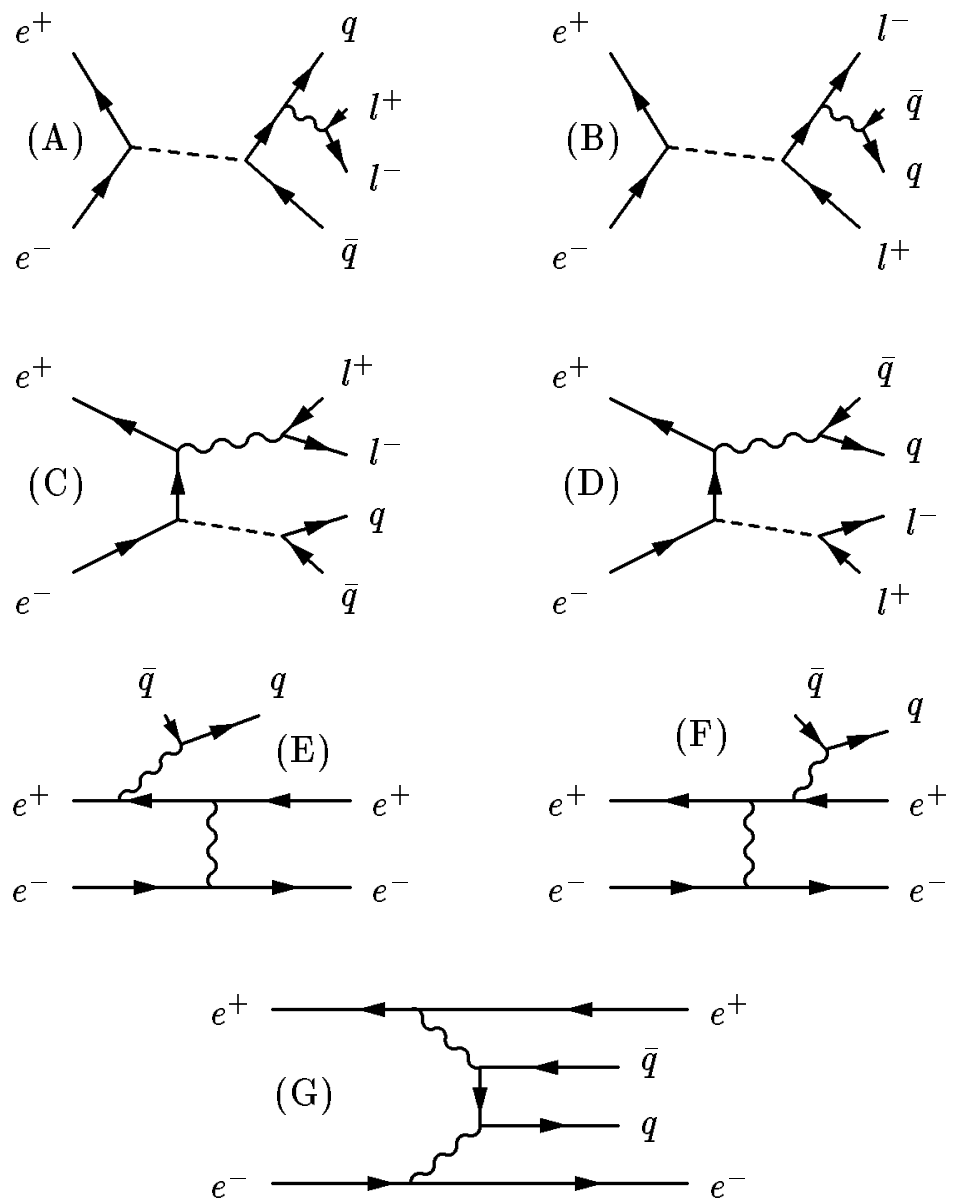

Figure 1: Schematic Feynman diagrams for the four-fermion production process $\mathrm{e}^{+} \mathrm{e}^{-} \rightarrow \ell^{+} \ell^{-} \mathrm{q} \overline{\mathrm{q}}$, divided into the four gauge-invariant groups: annihilation (A,B), conversion (C,D), bremsstrahlung $(\mathrm{E}, \mathrm{F})$ and multiperipheral $(\mathrm{G})$ diagrams. The dashed line symbolizes an intermediate $\gamma$ or $\mathrm{Z}$ boson. The contribution of the respective diagrams is especially high if the $\mathrm{Z}$ boson is on its mass shell. In addition all photon lines can also be replaced by a $\mathrm{Z}$. 

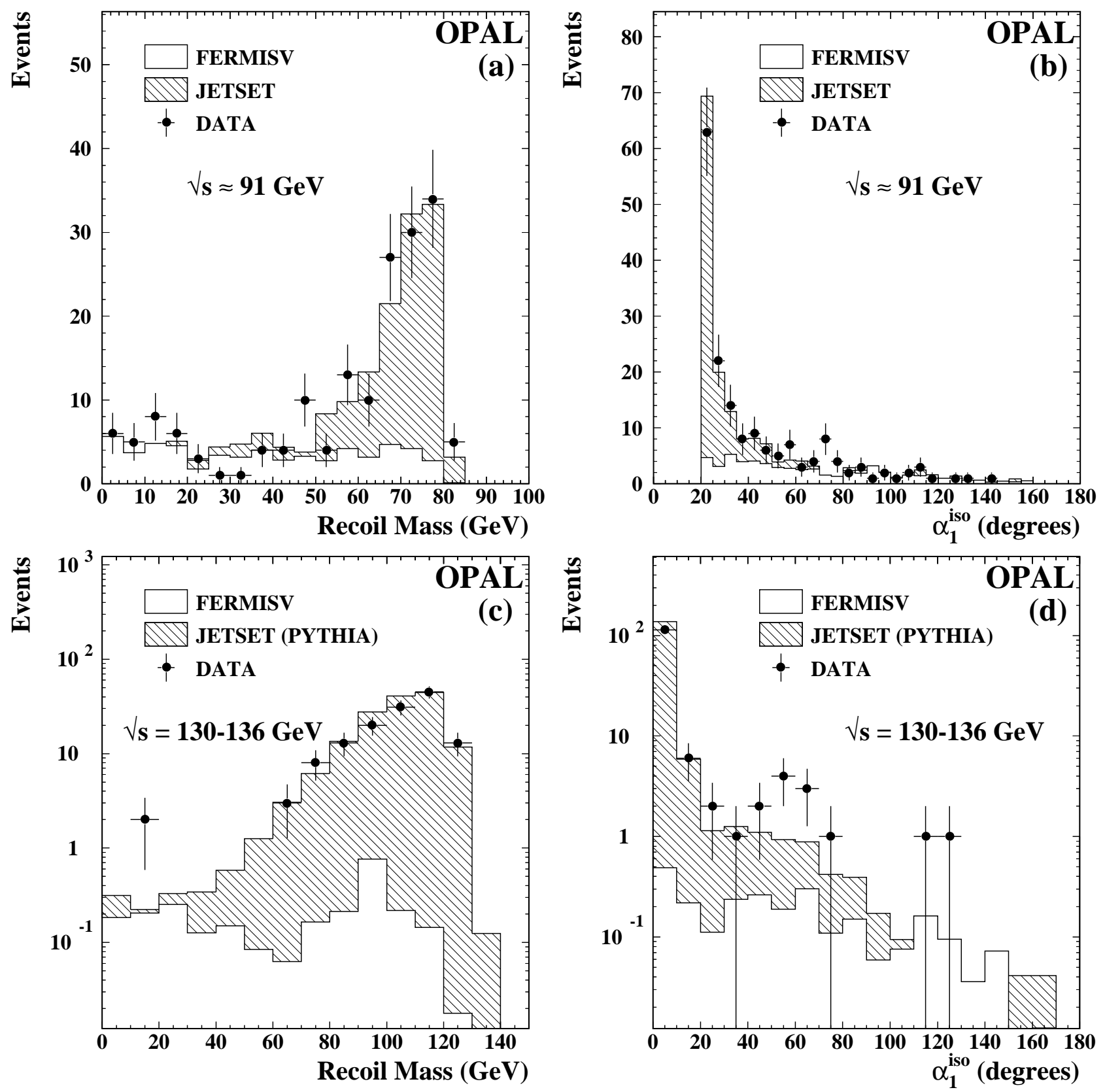

Figure 2: Recoil mass distribution (a,c) and distribution of $\alpha_{1}^{\text {iso }}(\mathrm{b}, \mathrm{d})$ for $\ell^{+} \ell^{-} \mathrm{q} \overline{\mathrm{q}}$ events at $\sqrt{s} \approx$ $m_{\mathrm{Z}}(\mathrm{a}, \mathrm{b})$ and $\sqrt{s}=130-136 \mathrm{GeV}(\mathrm{c}, \mathrm{d})$ after preselection. Points are the data, the open histogram is the FERMISV prediction and the hatched region is JETSET (PYTHIA) background. 

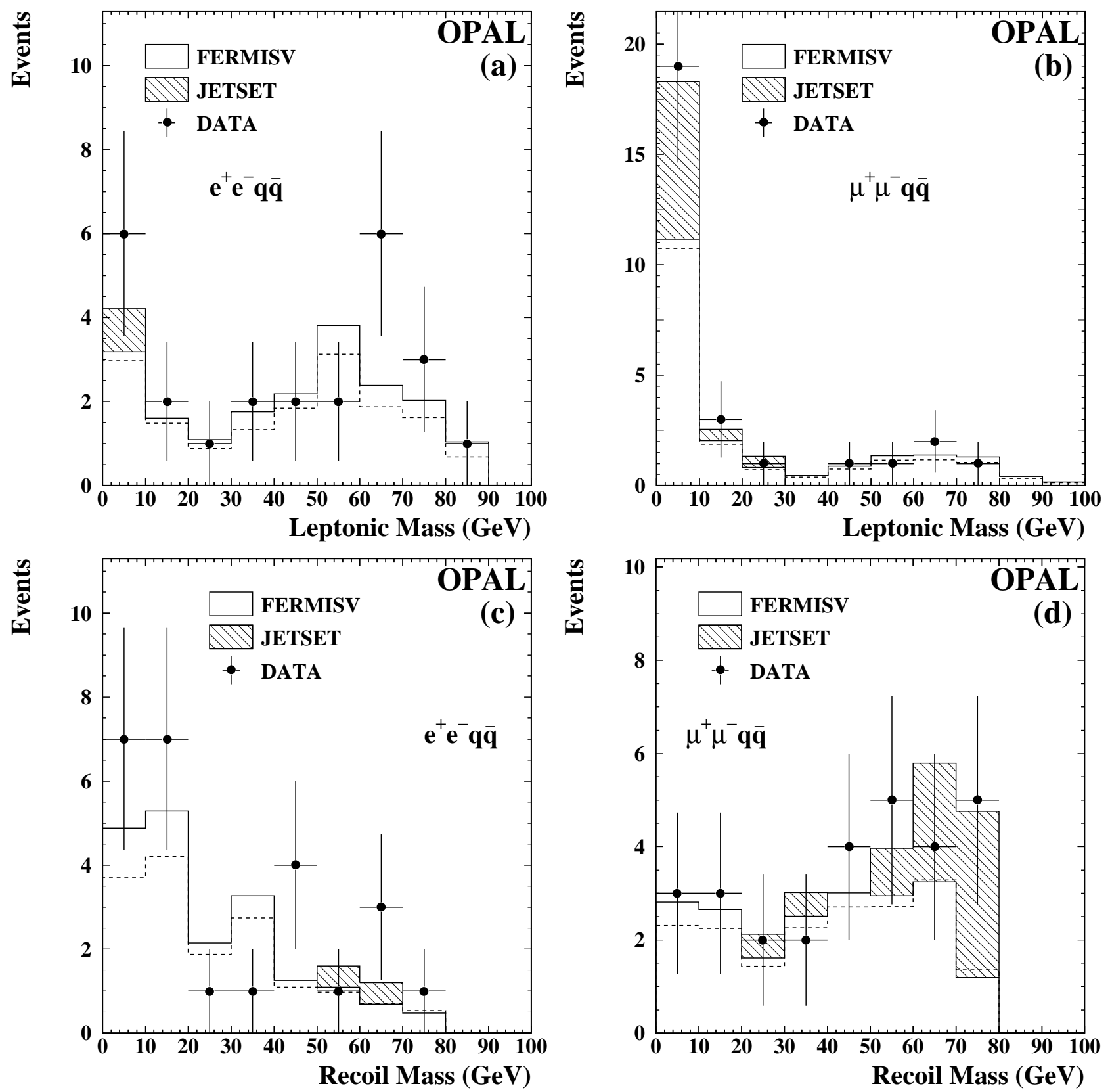

Figure 3: Leptonic invariant masses $(\mathrm{a}, \mathrm{b})$ and recoil masses $(\mathrm{c}, \mathrm{d})$ of the $\mathrm{e}^{+} \mathrm{e}^{-} \rightarrow \mathrm{e}^{+} \mathrm{e}^{-} \mathrm{q} \overline{\mathrm{q}}(\mathrm{a}, \mathrm{c})$ and $\mu^{+} \mu^{-} \mathrm{q} \overline{\mathrm{q}}(\mathrm{b}, \mathrm{d})$ channel for the final event selection at $\sqrt{s} \approx m_{\mathrm{Z}}$. Crosses are the data, the open histogram is the FERMISV prediction, and the hatched region is JETSET background. The dashed histogram shows the FERMISV prediction without the higher order corrections. 

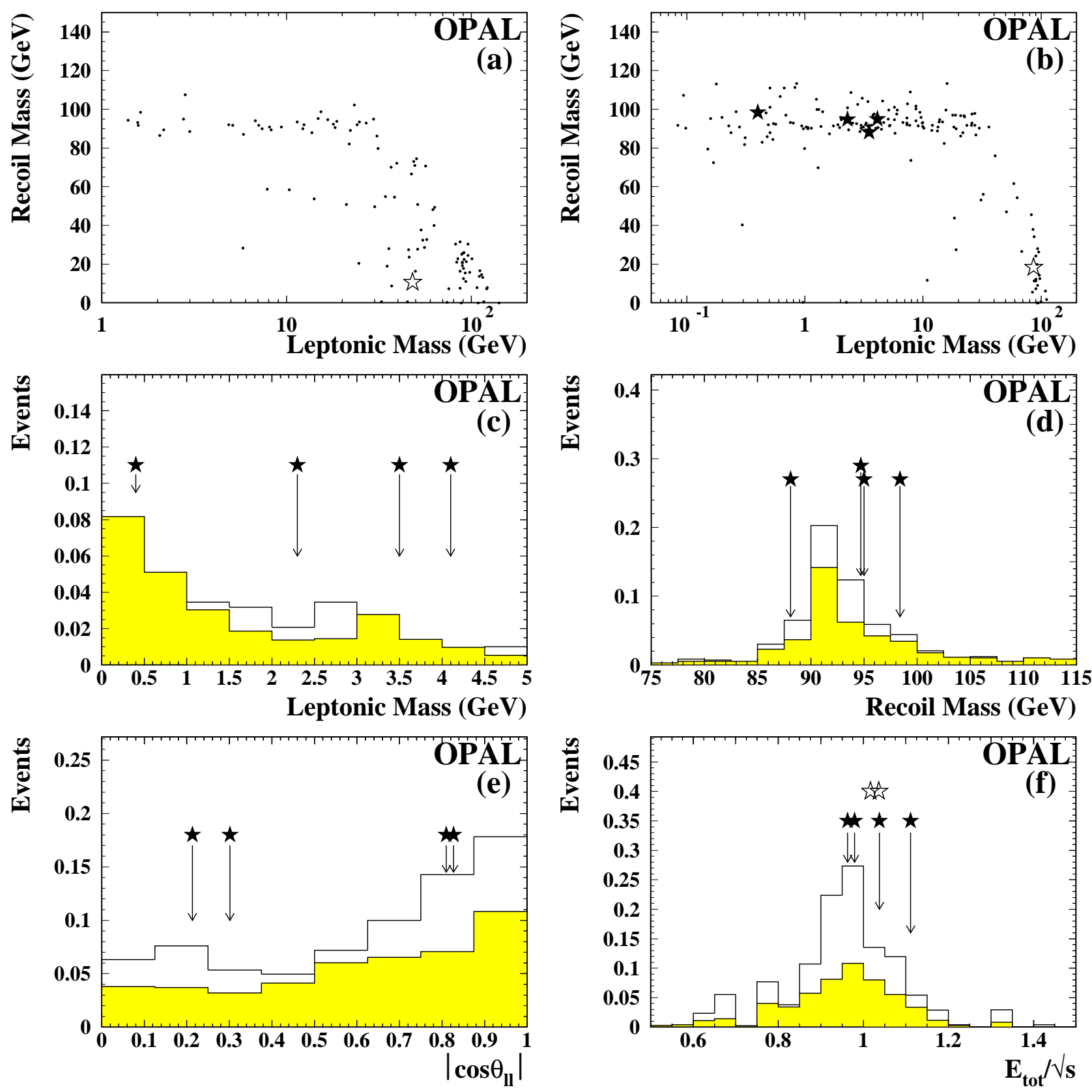

Figure 4: Recoil mass versus leptonic mass for the final event selection at $\sqrt{s}=130-136 \mathrm{GeV}$ for (a) $\mathrm{e}^{+} \mathrm{e}^{-} \mathrm{q} \overline{\mathrm{q}}$, and (b) $\mu^{+} \mu^{-} \mathrm{q} \overline{\mathrm{q}}$ events; the small dots are the FERMISV Monte Carlo prediction and the stars represent the 6 data events. (c)-(e) Various distributions for events with large recoil mass for $\mathrm{e}^{+} \mathrm{e}^{-} \mathrm{q} \overline{\mathrm{q}}$ (open histogram) and $\mu^{+} \mu^{-} \mathrm{q} \overline{\mathrm{q}}$ (shaded histogram); (c) leptonic mass, (d) recoil mass, (e) cosine of the angle of lepton momentum sum with respect to the beam axis. (f) $E_{\text {tot }} / \sqrt{s}$ : the scaled total visible energy for all events. In all histograms the values of the remaining high recoil mass $\left(\mu^{+} \mu^{-} \mathrm{q} \bar{q}\right)$ events are indicated by filled stars, while the open stars represent the events with low recoil mass. 